40th "Jaszowiec" International School and Conference on the Physics of Semiconductors, Krynica-Zdrój 2011

\title{
Hopping Excitons in GaInNAs - Simulation of Micro- and Macrophotoluminescence Spectra
}

\author{
M. Baranowski*, M. Latkowska, R. Kudrawiec and J. Misiewicz \\ Institute of Physics, Wrocław University of Technology, Wybrzeże Wyspiańskiego 27, 50-370 Wrocław, Poland
}

\begin{abstract}
Model of hopping excitons has been applied to explain the origin of sharp lines observed in microphotoluminescence spectra of GaInNAs layers. It has been shown that shape of the micro- and macrophotoluminescence spectra results from the exciton hopping between localizing centers and this phenomenon is responsible for the observation of sharp lines in microphotoluminescence experiment. In addition, the influence of different model parameters on photoluminescence spectra and their characteristic parameters such as the Stokes shift and full width at half maximum has been investigated.
\end{abstract}

PACS: 78.66.Fd, 78.55.Cr, 78.20.Bh

\section{Introduction}

The incorporation of small amount of nitrogen (few percent) into $\mathrm{Ga}(\mathrm{In})$ As host strongly affects its optical properties. The energy of emission is strongly reduced [1] and photoluminescence (PL) spectra became broadened and asymmetric. Additionally, temperature dependence of PL peak energy exhibits deviation from Varshni's formula i.e. so-called S-shape [2, 3]. The broadening, asymmetry and S-shape of PL peak are usually explained by the presence of localized states below the band gap. The origin of localizing states is connected with the presence of alloy content fluctuation and point defect induced by nitrogen incorporation. It has been shown in micro-PL experiment that in fact broad PL band observed at low temperature is composed of sharp lines [4-6], which are associated with the recombination of single excitons trapped by the localization potentials.

In papers $[7,8]$ it was shown that model of hopping excitons can explained phenomena observed in macro-PL spectra in diluted nitrides material. Recently we have shown that this model with some modification can be also applied to explain origin of sharp lines observed in micro-PL experiment [9]. Additionally, our modification allows us to reconstruct results of temperature and power dependent experiments [9]. In this report we want to show that hopping of excitons between different localizing states has major impact on observed sharp lines in micro-PL experiment. Additionally, we investigate the influence of different parameters of hopping excitons model on a generated micro- and macro-PL spectra.

\section{Theoretical model}

Model that we apply to simulate PL spectra is described in detail in paper [9], so here we just presents brief description. Our model takes into account recombination from localized states as well from delocalized

* corresponding author; e-mail: michal.baranowski@pwr.wroc.pl states. The rate of radiative recombination equals

$$
v_{\mathrm{r}}=\tau_{0}^{-1} \text {. }
$$

where $\tau_{0}$ is exciton lifetime. The rate of exciton activation above the mobility edge from a localized state $i$ with the energy $\varepsilon_{i}$ below the mobility edge is equal to

$$
v_{\mathrm{a}}=v_{0}\left(-\frac{\varepsilon_{i}}{k T}\right) \text {, }
$$

and the hopping rate from side $i$ to unoccupied side $j$ is calculated according to the Miller-Abrahams formula

$$
v_{i j}=v_{0}\left(-\frac{2 r_{i j}}{\alpha}-\frac{\varepsilon_{j}-\varepsilon_{i}+\left|\varepsilon_{j}-\varepsilon_{i}\right|}{2 k T}\right),
$$

where $\varepsilon_{i}$ is the energy of localized state, $\alpha$ is the decay length of exciton wave function and $v_{0}$ is the attempt to escape frequency. We assume that the rate of nonradiative recombination is described by the expression

$$
v_{\mathrm{nr}}=v_{\mathrm{d}} \exp \left(\frac{-\varepsilon_{\mathrm{b}}}{k T}\right)
$$

where the $v_{\mathrm{d}}$ is the rate of exciton dissociation (nonradiative recombination) and the $\varepsilon_{\mathrm{b}}$ is the binding energy of free excitons in GaInNAs. A free exciton can be captured by one of unoccupied localized center with the rate

$$
v_{\text {cap }}=v_{0} \frac{N_{\mathrm{f}}}{N},
$$

where $N_{\mathrm{f}}$ is a number of unoccupied localized states and $N$ is the total number of localized states. Proposed model takes into account saturation effects and simulation can be performed with different number of exctions created at the beginning. Free exciton recombines with some random energy which is described by the Gaussian distribution resulting from alloy inhomogeneities. For recombination from localized states we use the Lorentz line shape and we assume an exponential density of states (DOS) for localized states

$$
\operatorname{DOS}(\varepsilon)=\frac{N / L^{3}}{\epsilon_{0}} \exp \left(-\frac{\varepsilon}{\varepsilon_{0}},\right)
$$

where $N$ is the number of defects states and $L$ is the size 
of cubic volume where defects states are situated in a random way. $\epsilon_{0}$ is an average value of energy of defects states. The crucial effects on the observed spectrum have parameters $\tau_{0}, v_{0}$ and $\frac{N \alpha^{3}}{L^{3}}$.

\section{Simulation results}

Simulations were performed with according parameters value $\tau_{0} v_{0}=10^{3}$, Gaussian line broadening $13 \mathrm{meV}$ and the Lorentz line broadening $0.7 \mathrm{meV}$. Amount of defects states is $N=1000$ and the initial amount of injected carriers is equal to $n_{0}=500$. Temperature $T$ equals $5 \mathrm{~K}$. The simulations are repeated many times and averaged to make results reliable. The micro-PL simulations are repeated on a one localizing states population (one drawing of localizing states parameters). In case of macro-PL simulations the averaging is performed on a different localizing states population (many drawing) to achieve condition that are in experiment, where signal is collected from much bigger area of the sample. Simulations are performed for different $\frac{N \alpha^{3}}{L^{3}}$ and $\epsilon_{0}$ value to show influence of this parameter on generated spectra.

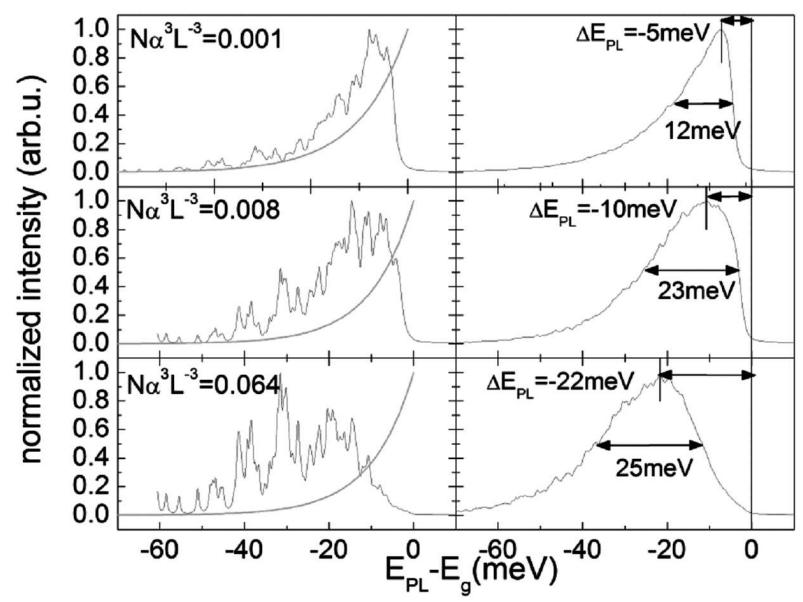

Fig. 1. Micro-PL (left side) and macro-PL spectra (right side) for different value of $\frac{N \alpha^{3}}{L^{3}}$, the $\varepsilon_{0}$ is equal to $10 \mathrm{meV}$. The gray line represents the DOS of localizing states.

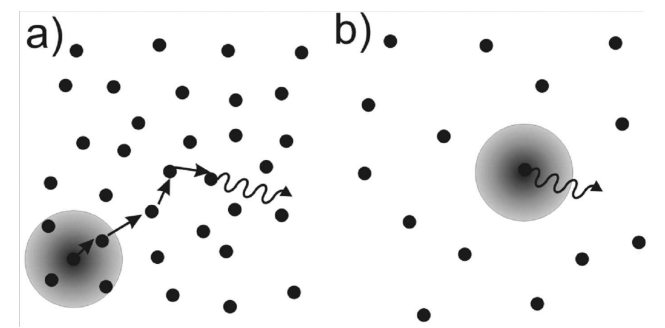

Fig. 2. Schema of exciton behavior for high (a) and low (b) value of $\frac{N \alpha^{3}}{L^{3}}$. Straight arrows mark hopping, waved arrow marks radiative recombination.
Figure 1 shows generated micro- and macro-PL spectra for $\epsilon_{0}=10 \mathrm{meV}$ and different $\frac{N \alpha^{3}}{L^{3}}$ value. On the left side there are micro-PL spectra and on the right side - macro-PL. We can see that with increasing value of $\frac{N \alpha^{3}}{L^{3}}$ the shape of micro- and macro-PL spectra start to change. For low value of $\frac{N \alpha^{3}}{L^{3}}$ the micro-PL spectrum is relatively smooth and quite well reproduces the density of localizing states which is marked by gray line, however when $\frac{N \alpha^{3}}{L^{3}}$ increases more, sharp features appear in the spectrum and shape of photoluminescence band does not reproduce the distribution of localizing states DOS given by Eq. (6). In the same time the micro- and macro-PL peak is redshifted (i.e. the Stokes shifts increases) with increasing $\frac{N \alpha^{3}}{L^{3}}$. Explanation of this fact is schematically presented in Fig. 2. When the $\frac{N \alpha^{3}}{L^{3}}$ ratio is large, then the probability of exciton hopping between different states is also large. In this case in the range of the decay length of exciton wave function $\alpha$ there are other localizing states and exciton can easily perform some hopping before the radiative recombination (Fig. 2a). If the probability of hopping is small then excitons usually recombine after the first capture process (Fig. 2b) and because of that photoluminescence reproduces the density of localizing states. For a high $\frac{N \alpha^{3}}{L^{3}}$ ratio PL band shifts to low energy side since the process of hopping to higher energy states is less probable than to lower energy states especially at low temperatures, which means that excitons relax to states with larger localization energy. Of course, when the $\frac{N \alpha^{3}}{L^{3}}$ ratio is small, then the redshift of PL is smaller because excitons do not perform many hoppings before recombination and they are not able to achieve deeply situated localizing states - excitons are mainly captured at the beginning by shallow localizing states because of the shape of DOS. Appearing of sharp lines with increasing $\frac{N \alpha^{3}}{L^{3}}$ is related to the random distribution of localized states. In such a system some states have such a neighbourhood, which makes the relaxation to this state very attractive. Excitons captured by localized centres from such a neighbourhood relax to this attractive state and after that they recombine in this state. When the $\frac{N \alpha^{3}}{L^{3}}$ ratio is small then the exciton hopping is frozen and there are no favourable localized states which could be manifested by sharp lines in micro-PL spectra. Summarizing this discussion, the well separated sharp lines observed in micro-PL spectra would not be observed without exciton hopping.

Figure 3 shows influence of $\varepsilon_{0}$ parameter on generated photoluminescence spectra. We can see that with increasing $\varepsilon_{0}$ the Stokes shift and full width at half maximum (FWHM) also increases. This behavior is not surprising, since $\varepsilon_{0}$ is an average value of localizing states energy. We can also see that when we increase value of $\varepsilon_{0}$, more sharp lines appear. For $\varepsilon_{0}=5 \mathrm{meV}$ there are only few sharp lines in micro-PL spectra while for $\varepsilon_{0}=15 \mathrm{meV}$ there are much more sharp lines. This is due to change of average energetic distance between localizing states. 


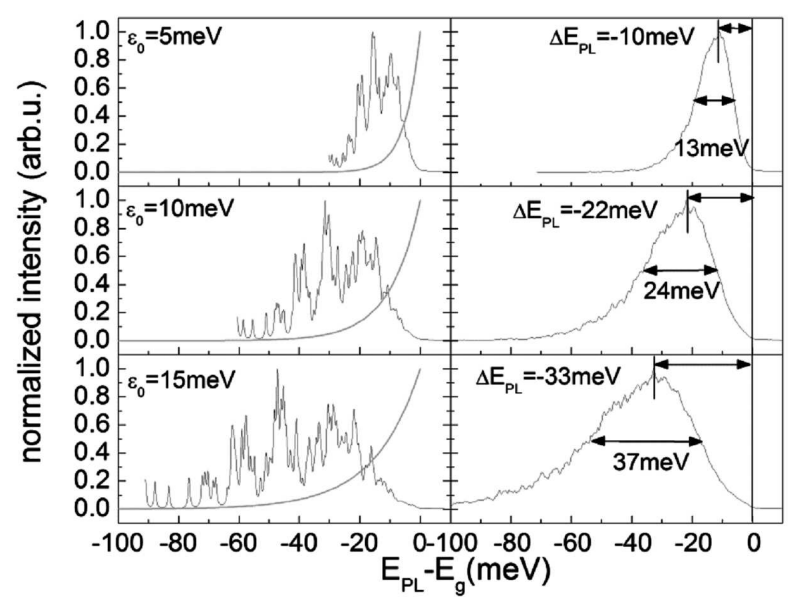

Fig. 3. Micro-PL (left side) and macro-PL spectra (right side) for different value of $\varepsilon_{0}$, the $\frac{N \alpha^{3}}{L^{3}}$ is equal to 0.064 . The gray line represents the DOS of localizing states.

When different localizing states have similar energy then emission from different localizing states is hard to resolve (every transition has some broadening). When $\varepsilon_{0}$ is big then emission from different localizing states can be easily resolved. Increase of average energy distance between localizing states also cause increase of FWHM.

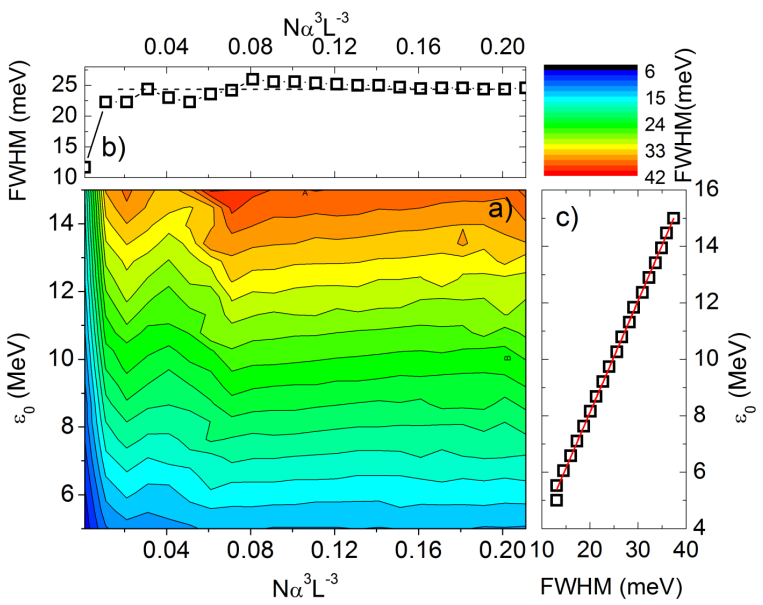

Fig. 4. (a) Dependence of FWHM versus $\frac{N \alpha^{3}}{L^{3}}$ and $\varepsilon_{0}$, (b) horizontal cross-section at $\varepsilon_{0}=10 \mathrm{meV}$, (c) vertical cross-section at $\frac{N \alpha^{3}}{L^{3}}=0.064$; red line is a linear fit, $\mathrm{FWHM}=2.5 \varepsilon_{0}$.

From Fig. 3 it is visible that with increasing $\varepsilon_{0}$ the value of FWHM increases monotonically. In the same time from Fig. 1 we can conclude that with increase of $\frac{N \alpha^{3}}{L^{3}}$ the initial FWHM increases rapidly but later this increase is not so significant. To be sure that this observation is not accidental we calculate FWHM for wide range of $\varepsilon_{0}$ and $\frac{N \alpha^{3}}{L^{3}}$. Results of this calculation are presented in Fig. 4a. From this map we can see that FWHM is affected by $\frac{N \alpha^{3}}{L^{3}}$ only for the very small value of $\frac{N \alpha^{3}}{L^{3}}$. This is clearly visible on horizontal cross-section taken at $\varepsilon_{0}=10 \mathrm{meV}$, see Fig. $4 \mathrm{~b}$. For the value of $\frac{N \alpha^{3}}{L^{3}}$ higher than 0.01 the value of FWHM is nearly constant and equal to about $24 \mathrm{meV}$. In the same time FWHM rises linearly with $\varepsilon_{0}$ (Fig. 4c) and the coefficient of proportionality is equal to about 2.5. This means that FWHM is affected mainly by the average energy of localizing states and it does not depend on chosen $\frac{N \alpha^{3}}{L^{3}}$. This observation gives us an easy method for estimation of $\varepsilon_{0}$ from experimental data.

\section{Summary}

In summary we have shown that according to proposed model process of excitons hopping is crucial for sharp lines to be observed in micro-PL experiment and shape of photoluminescence spectra. We have also shown that $\frac{N \alpha^{3}}{L^{3}}$ and average energy affect the shape of micro-PL and macro-PL spectra. It has been found that the Stokes shift of PL is affected by both $\frac{N \alpha^{3}}{L^{3}}$ and $\varepsilon_{0}$, while FWHM is affected only by the $\varepsilon_{0}$. The dependence of FWHM on $\varepsilon_{0}$ is linear. It means that FWHM can be used as parameter for estimation of $\varepsilon_{0}$ from experimental data.

\section{Acknowledgments}

The authors acknowledge support from the MNiSW (grant no. N202 258339).

\section{References}

[1] W. Shan, W. Walukiewicz, J.W. Ager, E.E. Haller, J.F. Geisz, D.J. Friedman, J.M. Olson, S.R. Kurtz, Phys. Rev. Lett. 82, 1221 (1999).

[2] I.A. Buyanova, W.M. Chen, G. Pozina, J.P. Bergman, B. Monemar, H.P. Xin, C.W. Tu, Appl. Phys. Lett. 75, 501 (1999).

[3] A. Kaschner, T. Lüttgert, H. Born, A. Hoffmann, A.Yu. Egorov, H. Riechert, Appl. Phys. Lett. 78, 1391 (2001).

[4] R. Kudrawiec, G. Sęk, J. Misiewicz, F. Ishikawa, A. Trampert, K.H. Ploog, Appl. Phys. Lett. 94, 011907 (2009).

[5] M. Latkowska, R. Kudrawiec, G. Sęk, J. Misiewicz, J. Ibáñez, M. Henini, M. Hopkinson, Phys. Status Solidi C 8, 1655 (2011).

[6] M. Latkowska, R. Kudrawiec, G. Sęk, J. Misiewicz, J. Ibáñez, M. Henini, M. Hopkinson, Appl. Phys. Lett. 98, 131903 (2011).

[7] S.D. Baranovskii, R. Eichmann, P. Thomas, Phys. Rev. B 58, 13081 (1998).

[8] O. Rubel, S.D. Baranovskii, K. Hantke, B. Kunert, W.W. Rühle, P. Thomas, K. Volz, W. Stolz, Phys. Rev. B 73, 233201 (2006).

[9] M. Baranowski, M. Latkowska, R. Kudrawiec, J. Misiewicz, J. Phys., Condens. Matter 23, 205804 (2011). 